\title{
RESEARCH
}

Open Access

\section{The circadian rest-activity pattern predicts cognitive decline among mild-moderate Alzheimer's disease patients}

Adriano D. S. Targa ${ }^{1,2+}{ }^{4}$ Iván D. Benítez ${ }^{1,2 \dagger}{ }^{2}$, Faridé Dakterzada ${ }^{3}$, John Fontenele-Araujo ${ }^{4}$, Olga Minguez',

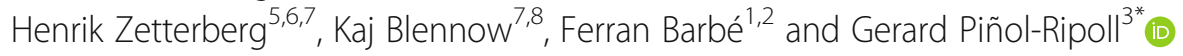

\begin{abstract}
Background: Alterations in circadian rhythms are present in the presymptomatic stage of Alzheimer's disease (AD), possibly contributing to its pathogenesis. However, it is unknown whether such alterations are associated with worse outcomes once individuals are diagnosed with symptomatic disease. We aimed to evaluate the association between the circadian rest-activity pattern and AD-related features in patients with mild-moderate AD.

Methods: We assessed the circadian rest-activity pattern of consecutive patients with mild-moderate AD through actigraphy for 14 days. Cerebrospinal fluid was obtained to determine the levels of important pathological markers including amyloid-beta protein (Aß42), phosphorylated tau (P-tau), total tau (T-tau), and neurofilament light (NF-L). Neuropsychological evaluation was conducted at the beginning of the study and after 12 months of follow-up. Linear regression models were performed considering the global population and $A \beta 42+$ patients only.

Results: The cohort included 100 patients with mild-moderate AD. The median age $\left[p_{25} ; p_{75}\right]$ was 76.0 [73.0;80.0] years and 63.0\% were female. Older age (effect size [SE] of 0.324 [0.096]; $p=0.001)$ and male sex $(0.780$ [0.193]; $p=$ 0.001 ) were associated with increased fragmentation and decreased synchronization of the rhythm, respectively. After adjusting for age, sex, and season of the year, increased levels of T-tau (effect size [95\% Cl] of 0.343 [0.139 to $0.547] ; p=0.001)$ and NF-L (0.444 [0.212 to 0.676$] ; p=0.001)$ were associated with a higher amplitude of the restactivity rhythm. Increased fragmentation of the rhythm at baseline was associated with greater cognitive decline after one year of follow-up independent of age, sex, T-tau/A 342 ratio, educational level, and season of the year (0.715 [- 1.272 to -0.157$] ; p=0.013$ ). Similar findings were obtained considering only the A $342+$ patients.

Conclusions: Our results suggest a potential role of the circadian rest-activity pattern in predicting the cognitive decline of patients with mild-moderate AD. Further studies are warranted to confirm these findings and to elucidate whether there is causality among the observed associations.
\end{abstract}

Keywords: Alzheimer's disease, Circadian rest-activity pattern, Intradaily variability, Neurofilament light, Cognitive decline

\footnotetext{
* Correspondence: gerard_437302@hotmail.com

${ }^{\dagger}$ Adriano D. S. Targa and Iván D. Benítez are co-first authors and contributed equally to this study.

${ }^{3}$ Unitat Trastorns Cognitius, Clinical Neuroscience Research, Hospital Universitari Santa Maria, IRBLleida, Lleida, Spain

Full list of author information is available at the end of the article
}

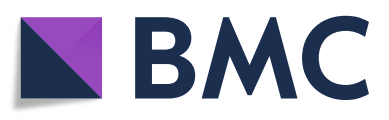

(c) The Author(s). 2021 Open Access This article is licensed under a Creative Commons Attribution 4.0 International License, which permits use, sharing, adaptation, distribution and reproduction in any medium or format, as long as you give appropriate credit to the original author(s) and the source, provide a link to the Creative Commons licence, and indicate if changes were made. The images or other third party material in this article are included in the article's Creative Commons licence, unless indicated otherwise in a credit line to the material. If material is not included in the article's Creative Commons licence and your intended use is not permitted by statutory regulation or exceeds the permitted use, you will need to obtain permission directly from the copyright holder. To view a copy of this licence, visit http://creativecommons.org/licenses/by/4.0/ The Creative Commons Public Domain Dedication waiver (http://creativecommons.org/publicdomain/zero/1.0/) applies to the data made available in this article, unless otherwise stated in a credit line to the data. 


\section{Introduction}

Alzheimer's disease (AD) is the most prevalent neurodegenerative disorder in the world and currently affects nearly 50 million people $[1,2]$. This number is expected to increase given the age-associated risk and the growing number of older adults [2-4]. The pathological hallmarks of $\mathrm{AD}$ are the deposition of amyloid-beta protein (A $\beta 42)$, the formation of tau protein neurofibrillary tangles, and neurodegeneration [5]. Symptomatically, there is a progressive loss of cognitive function, which is often concomitant with behavioral symptoms, including depression, anxiety, hallucinations, sleep disturbances, and alterations in circadian rhythms [6-8].

Circadian rhythm alterations are mainly represented by the loss of a well-defined 24-h rest-activity pattern due to increased activity during the night and a decrease in activity during the day $[9,10]$. Accordingly, sleep fragmentation and the presence of irregular bouts of sleep during the day decrease the amplitude and increase the fragmentation of the rest-activity rhythm [11, 12]. In addition, shifts in the bedtime and wake-up time to a later time in the day (i.e., phase delay) may occur in contrast to the usual advance of the phase observed in older adults $[13,14]$. Furthermore, other circadian alterations can be observed, including those related to the secretion patterns of hormones such as melatonin and cortisol as well as in the daily fluctuations of body core temperature [15-17].

Alterations in circadian rhythms may precede the onset of classical cognitive symptoms in $\mathrm{AD}$ patients. A previous study demonstrated that cognitively unimpaired individuals with preclinical amyloid plaque pathology presented increased fragmentation of rest-activity rhythm independent of age and sex [10]. This suggests that circadian alterations could be used as biomarkers for the preclinical stage and/or contribute to the pathogenesis of the disease. Accordingly, prospective studies appear to support the idea that these alterations increase the risk of cognitive deterioration, leading to mild cognitive impairment and dementia [18-21]. Li and colleagues demonstrated that lower amplitude and higher fragmentation of the rest-activity rhythm increased the risk of developing $\mathrm{AD}$ in healthy older adults [8]. Similarly, these dysfunctions in addition to decreased interdaily stability independently increased the risk of such outcomes in individuals previously diagnosed with mild cognitive impairment [8]. However, it is unknown whether circadian alterations could be associated with worse outcomes once individuals have symptomatic AD.

To investigate this, we first performed a comprehensive characterization of the circadian rest-activity pattern of mild-moderate AD patients. In the sequence, we investigated whether the rest-activity rhythm could be associated with biomarkers of $\mathrm{AD}$, such as $\mathrm{A} \beta 42$, phosphorylated tau
(P-tau), total tau (T-tau), and neurofilament light (NF-L). Finally, we evaluated whether the rest-activity rhythm could predict the cognitive decline of mild-moderate AD patients at 12 months of follow-up.

\section{Methods \\ Study population}

This is an ancillary study of a prospective trial designed to evaluate the influence of obstructive sleep apnea (OSA) on the cognitive decline of AD patients after one year of follow-up (NCT02814045). Patients were recruited at the Cognitive Disorders Unit of the Hospital Universitari Santa Maria (Spain) for 4 years (2015-2019). The inclusion criteria comprised acetylcholinesterase inhibitor-naïve individuals aged over 60 years who were diagnosed with $\mathrm{AD}$ according to the National Institute on Aging and Alzheimer's Association (NIA-AA) clinical criteria [22]. Accordingly, to include patients with mildmoderate cognitive impairment, we only considered those with a Mini-Mental State Examination (MMSE) score $\geq 20$. Secondary analyses were performed only with the patients who were $\mathrm{A} \beta 42+$ (values $<600 \mathrm{pg} / \mathrm{ml}$ were considered pathological amyloid deposition) or A 342 - and had an MMSE score $\geq 20$.

The exclusion criteria comprised (1) the presence of visual and/or communication problems that could make compliance with the study procedures difficult; (2) the presence of a previously diagnosed sleep disturbance; (3) the presence of excessive somnolence for unknown reasons; (4) comorbidities such as cancer, severe renal or hepatic insufficiency, severe cardiac or respiratory failure; (5) excessive alcohol intake (> 280 g/week); (6) MRI evidence of hydrocephalus, stroke, a space-occupying lesion, or any clinically relevant central nervous system disease other than $\mathrm{AD}$; (7) the presence of mental disorders according to DSM-V-TR ${ }^{\mathrm{TM}}$ criteria; (8) any use of medications under investigation; (9) the presence of untreated (or treated for less than 3 months prior to the screening visit) vitamin B12 or folate deficiency; and (10) the presence of untreated thyroid disease.

The study was approved by the care ethics committee of Hospital Universitari Arnau de Vilanova (CE-1218) and conducted according to the Declaration of Helsinki. The patient, the responsible caregiver, and the legal representative (when different from the responsible caregiver) signed an informed consent form.

\section{Study design}

After arriving at the Cognitive Disorders Unit of the Hospital Universitari Santa Maria (Spain), eligible patients were subjected to clinical evaluation for anthropometric data and sociodemographic data collection. Blood was obtained to determine apolipoprotein $\mathrm{E}$ (ApoE) genotypes, and cerebrospinal fluid (CSF) was collected to 
determine the levels of Aß42, T-tau, P-tau, and NF-L. In the sequence, the patients received a sleep log to be completed over 14 days as well as instructions related to the use of the actigraph that occurred during the same period. The neuropsychological evaluation was performed at the beginning of the study and after 12 months of follow-up.

\section{Clinical variables}

The following variables were collected: age, sex, years of education, alcohol consumption, smoking, vascular risk factors (hypertension, diabetes mellitus, dyslipidemia, stroke, and cardiopathy), personal psychiatric history, and family psychiatric and neurological history. The body mass index (BMI) was calculated as body weight (in $\mathrm{kg}$ )/height (in $\mathrm{m}^{2}$ ).

\section{Apolipoprotein E (ApoE) genotype}

DNA was extracted from buffy coat cells using a Maxwell ${ }^{\circ}$ RCS blood DNA kit (Promega, USA). Twenty microliters of DNA were used for ApoE genotyping by polymerase chain reaction (PCR). ApoE genotype was dichotomized as ApoE- $\varepsilon 4$ homozygous or heterozygous

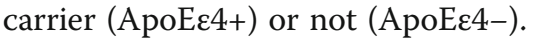

\section{CSF biomarkers}

The CSF samples were collected at baseline between 8:00-10:00 a.m. They were placed in polypropylene tubes, centrifuged at $2000 \times g$ for $10 \mathrm{~min}$ at $4{ }^{\circ} \mathrm{C}$, immediately frozen, and stored within $4 \mathrm{~h}$ in a $-80^{\circ} \mathrm{C}$ freezer. The measurement of Aß42, T-tau, and P-tau was performed using commercial kits (Innotest $^{\circ} \beta$ Amyloid-42; Innotest $^{\circ}$ hTAU Ag; and Innotest ${ }^{\circ}$ Phospho-TAU181P, Fujirebio-Europe, Gent, Belgium). NF-L was measured by a commercial ELISA kit (Quidel, San Diego, CA). All determinations were performed in duplicate and in one round of experiments using one batch of reagents by board-certified laboratory technicians who were blinded to the clinical data. The intra-assay coefficients of variation were lower than $10 \%$ for internal quality control samples (two per plate). Based on previous data obtained by the research group, A 442 levels $<600 \mathrm{pg} / \mathrm{ml}$ were considered pathological [23].

\section{Circadian rest-activity pattern assessment}

Rest-activity data were collected using a wrist-mounted actigraph (Actiwatch 2, Philips Respironics) for 14 days. Activity counts of 30-s epochs with a medium threshold for sensitivity were obtained and visualized using a software (Actiware 6.0.9, Philips Respironics). The data were revised with the support of a sleep log which was completed during the 14 days of actigraphy.
The following variables were obtained: time in bed (in minutes), total sleep time (in minutes), sleep efficiency (in \%, defined as the ratio between total sleep time and the time spent in bed), latency (in minutes, defined as the time spent awake until the first sleep episode, which was represented by the first epoch of 10-min continuous stretch of immobility), and WASO (in minutes, defined as the time spent awake after sleep onset). In addition, different variables associated with the rest-activity rhythm were calculated from the 30-s epochs activity counts using the $\mathrm{R}$ software (version 3.4.2) $[24,25]$. The intradaily variability represented the fragmentation of the rest-activity rhythm within each 24-h period, indicating whether there were daytime naps and/or nocturnal activity episodes. The interdaily stability represented how similar one 24-h period was to the next, indicating how synchronized the internal rest-activity rhythm was with the different zeitgebers (24-h light-dark cycle, food intake, physical activity) along the 14 days of actigraphy. L5 (the mean activity of the five consecutive hours with the lowest activity) and M10 (the mean activity of the ten consecutive hours with the highest activity) were used to calculate the relative amplitude (M10-L5/ M10+L5). The relative amplitude represented the robustness of the rest-activity rhythm, indicating whether there was a difference in the magnitude of activity between active and rest phases.

\section{Neuropsychological assessment}

Patients underwent a neuropsychological evaluation through the MMSE at the beginning of the study and after 12 months of follow-up. The MMSE includes questions to evaluate different domains, such as attention, time and place orientation, and word recall. The scores of this test range from 0 to 30 , and a higher score indicates better cognitive function [26, 27].

\section{Statistical analysis}

Descriptive statistics were performed to describe sociodemographic, clinical, and AD-related data. The absolute and relative frequencies were used for qualitative data and the medians (25th percentile; 75th percentile $\left.\left[\mathrm{p}_{25} ; \mathrm{p}_{75}\right]\right)$ were estimated for quantitative variables. The normality of the distribution was assessed by the Shapiro-Wilk test. Intradaily variability and biomarkers values were log-transformed to normalize their distribution. The Bulging Rule transformation was used to normalize the relative amplitude [28].

The associations between rest-activity rhythm variables and sociodemographic, clinical, and sleep data were evaluated using linear models adjusted by age and sex. These analyses were performed including the global 
population. The associations between rest-activity rhythm variables and AD-related biomarkers were evaluated using linear models adjusted by age, sex, and season of the year [29]. These analyses were performed with the global population and stratified by amyloid groups (A $\beta 42+$ and $A \beta 42-$ patients). Additional analyses were performed to evaluate the possible effect of sex on this context [30,31]. The linear models were adjusted by age and season of the year and included the global population stratified by sex.

To investigate the association between circadian restactivity pattern and cognitive decline, we performed individual linear models including each variable of the rest-activity rhythm (interdaily stability, intradaily variability, and relative amplitude) as predictors and the MMSE at 12 months of follow-up as the outcome. The models were adjusted by age, sex, T-tau/A $\beta 42$ ratio, educational level, and season of the year. The analyses were performed with the global population and stratified by amyloid groups (A $\beta 42+$ and $A \beta 42$ - patients). Additional analyses were performed to evaluate the possible effect of sex. The linear models were adjusted by age, T-tau/ A 342 ratio, educational level, and season of the year, and included the global population stratified by sex. The residuals of the models were evaluated for their suitability in relation to the assumptions of the linear regression.

The $p$ value threshold defining statistical significance was set at $<0.05$. Data management and statistical analyses were performed using $\mathrm{R}$ (version 4.0.1).

\section{Results}

\section{Baseline characteristics}

The cohort included 100 patients diagnosed with mildmoderate $\mathrm{AD}$ with a median $\left[\mathrm{p}_{25} ; \mathrm{p}_{75}\right]$ of 23.5 [22.0;25.0] on the MMSE (Table 1). The median age was 76.0 [73.0; 80.0] years and $63.0 \%$ of the patients were female. Common comorbidities were hypertension (59.0\%), depression (28.0\%), cardiopathy (21.0\%), and diabetes mellitus (20.0\%). In addition, 41 (41.8\%) patients were ApoEs4+. Considering the most recent NIA-AA research framework based on the pathological process [32], we performed additional analyses including only the $\mathrm{A} \beta 42+$ patients. Similar characteristics were observed in this subpopulation (Additional file 1).

\section{Circadian rest-activity pattern and sleep}

We calculated the interdaily stability, intradaily variability, and relative amplitude to evaluate the rest-activity rhythm of our population (Table 2). This analysis demonstrated a median $\left[\mathrm{p}_{25} ; \mathrm{p}_{75}\right]$ of $0.57[0.49 ; 0.63]$ for interdaily stability, $0.80[0.68 ; 0.95]$ for intradaily variability, and $0.88[0.84 ; 0.92]$ for the relative amplitude. Most of the population $(56.0 \%)$ slept less than $7 \mathrm{~h}$ per night, with a median of $83.5 \%[78.1 ; 88.4]$ of sleep efficiency.
Table 1 Baseline characteristics of the cohort

\begin{tabular}{|c|c|}
\hline & $\begin{array}{l}\text { Global } \\
n=100 \\
n(\%) \text { or median }\left[p_{25} ; p_{75}\right]\end{array}$ \\
\hline \multicolumn{2}{|l|}{ Sociodemographic data } \\
\hline \multicolumn{2}{|l|}{ Sex } \\
\hline Female & $63(63.0 \%)$ \\
\hline Male & 37 (37.0\%) \\
\hline Age, years & $76.0[73.0 ; 80.0]$ \\
\hline $\mathrm{BMI}, \mathrm{kg} \cdot \mathrm{m}^{-2}$ & $27.6[25.1 ; 30.6]$ \\
\hline Education, years & $7.00[7.00 ; 7.00]$ \\
\hline \multicolumn{2}{|l|}{ Comorbidities } \\
\hline Hypertension & $59(59.0 \%)$ \\
\hline Diabetes mellitus & $20(20.0 \%)$ \\
\hline Cardiopathy & $21(21.0 \%)$ \\
\hline Depression & $28(28.3 \%)$ \\
\hline Periodic limb movements & $44(49.4 \%)$ \\
\hline \multicolumn{2}{|l|}{ Cognition } \\
\hline MMSE & $23.5[22.0 ; 25.0]$ \\
\hline \multicolumn{2}{|l|}{ CSF biomarkers } \\
\hline $\mathrm{A} \beta 42, \mathrm{pg} / \mathrm{ml}$ & $533[402 ; 669]$ \\
\hline T-tau, pg/ml & $464[330 ; 598]$ \\
\hline P-tau, pg/ml & $72.3[53 ; 93]$ \\
\hline \multicolumn{2}{|l|}{ Genetic risk } \\
\hline ApoEع4- & $41(41.8 \%)$ \\
\hline
\end{tabular}

Accordingly, there was a median of 39.4 [32.2;52.2] min of WASO, with $80 \%$ of the patients spending more than 30 min awake after sleep onset. The circadian restactivity pattern and the sleep of both subpopulations (A $\beta 42+$ and $A \beta 42$ - patients) presented similar characteristics to the global population (Additional file 2).

\section{Baseline characteristics and the circadian rest-activity pattern}

Linear regression models were performed to evaluate the baseline characteristics according to the rest-activity rhythm (Fig. 1). We observed an association between increased intradaily variability and higher age with an effect size (SE) of 0.324 (0.096), while increased interdaily stability was associated with female sex with an effect size of 0.780 (0.193). After adjusting for age and sex, there were significant associations between relative amplitude and sleep parameters with an effect size of 0.489 (0.088) for total sleep time, of $0.508(0.085)$ for sleep efficiency, and of $-0.590(0.080)$ for WASO. 
Table 2 Circadian rest-activity pattern and sleep

\begin{tabular}{|c|c|}
\hline & $\begin{array}{l}\text { Global } \\
n=100 \\
n(\%) \text { or median }\left[p_{25} ; p_{75}\right]\end{array}$ \\
\hline \multicolumn{2}{|l|}{ Rest-activity rhythm } \\
\hline Interdaily stability & $0.57[0.49 ; 0.63]$ \\
\hline Intradaily variability & $0.80[0.68 ; 0.95]$ \\
\hline Relative amplitude & $0.88[0.84 ; 0.92]$ \\
\hline M10 & $116[83.6 ; 151]$ \\
\hline L5 & $6.44[4.48 ; 9.93]$ \\
\hline \multicolumn{2}{|l|}{ Sleep } \\
\hline Time in bed, hours & $8.98[8.08 ; 9.57]$ \\
\hline Total sleep time, hours & $6.70[5.54 ; 7.56]$ \\
\hline$<7 \mathrm{~h}$ & $56(56.0 \%)$ \\
\hline $7-9 h$ & $39(39.0 \%)$ \\
\hline$>9 h$ & $5(5.0 \%)$ \\
\hline Sleep efficiency, \% & $83.5[78.1 ; 88.4]$ \\
\hline$\geq 85 \%$ & $44(44.0 \%)$ \\
\hline $84-75 \%$ & $41(41.0 \%)$ \\
\hline$<75 \%$ & $15(15.0 \%)$ \\
\hline Latency, minutes & $22.0[13.8 ; 33.2]$ \\
\hline$\leq 30 \mathrm{~min}$ & $69(69.0 \%)$ \\
\hline $31-60 \mathrm{~min}$ & $25(25.0 \%)$ \\
\hline$>60 \mathrm{~min}$ & $6(6.0 \%)$ \\
\hline WASO, minutes & $39.4[32.2 ; 52.2]$ \\
\hline$\leq 30 \min$ & $20(20.0 \%)$ \\
\hline$>30 \mathrm{~min}$ & $80(80.0 \%)$ \\
\hline
\end{tabular}

L5 the mean activity of the five consecutive hours with the lowest activity, M10 the mean activity of the ten consecutive hours with the highest activity, $n$ number, $p$ percentile, WASO time spent awake after sleep onset

\section{CSF biomarkers and the circadian rest-activity pattern}

Linear regression models adjusted for confounding factors (age, sex, and season of the year) were performed to assess the levels of the CSF biomarkers according to the rest-activity rhythm (Table 3 and Additional file 3 ). We observed that increased NF-L levels were associated with higher interdaily stability with an effect size [95\% CI] of 0.322 [0.076 to 0.569 ], 0.309 [ -0.003 to 0.620 ], and 0.603 [ -0.118 to 1.088 ] in the analyses considering the global population, $A \beta 42+$ patients, and $A \beta 42$ - patients, respectively. In addition, increased NF-L levels were associated with higher relative amplitude among the global population and $\mathrm{A} \beta 42+$ patients (global: 0.444 [0.212 to 0.676]; $A \beta 42+$ patients: 0.503 [0.209 to 0.797]), but the effect size was reduced among A $\beta 42-$ patients (0.186 [0.330 to 0.701$]$ ). Higher levels of T-tau were associated with increased relative amplitude in the three populations (global: 0.343 [0.139 to 0.547]; A $\beta 42+$ patients: 0.304 [0.046 to 0.561$]$; $A \beta 42-$ patients: 0.507 [0.124 to
0.890]). Additional analyses revealed similar results between males and females (Additional file 4).

\section{Cognitive decline and the circadian rest-activity pattern} We performed linear regression models to assess the cognitive decline according to the rest-activity rhythm (Table 4). A higher intradaily variability predicted an increased cognitive decline at one year of follow-up (effect size [95\% CI] of -0.715 [-1.272 to -0.157]) after adjusting for age, sex, T-tau/A $\beta 42$ ratio, educational level, and season of the year (Fig. 2). The analysis including only A $\beta 42+$ patients presented a similar effect size $(-0.717$ [- 1.391 to -0.042$])$, which was not maintained when considering only A $\beta 42-$ patients (- 0.579 [- 2.054 to 0.895]) (Additional files 5 and 6). Additional analyses suggested an effect of the sex within this context (Additional file 7). No associations were observed in relation to the interdaily stability and relative amplitude.

\section{Discussion}

In the current study, we performed a comprehensive evaluation of the circadian rest-activity pattern of patients with mild-moderate AD. The analysis demonstrated an association between older age and increased fragmentation of the rhythm. Female sex was associated with higher interdaily stability, suggesting increased synchronization between the endogenous rhythm and the zeitgebers in this subgroup of patients. Furthermore, fragmentation of the rhythm at baseline was associated with greater cognitive decline after one year of followup, suggesting a predictive role of the rest-activity pattern for the cognition of mild-moderate AD patients. Finally, we evaluated whether the rest-activity pattern was associated with different markers related to the disease's physiopathology, including A $\beta 42$, P-tau, T-tau, and NFL. This analysis revealed associations especially related to the relative amplitude.

The increased fragmentation of the rest-activity rhythm in older adults was previously demonstrated in healthy individuals $[10,33,34]$ and in subjects with diabetic complications [35], cancer [36], heart failure [37], and $\mathrm{AD}$ pathology without clinical symptoms [10]. In this study, we demonstrated that a similar relationship is also present in patients with mild-moderate AD. In contrast, we did not observe an association between interdaily stability and age. Several studies show divergent findings in this regard, reporting higher [33, 34], lower [8], or unchanged [10] synchronization between the endogenous rhythm and the zeitgebers in older adults, which may vary according to different factors such as institutionalization, retirement, and the presence of chronic diseases. There was an association between higher interdaily stability and female sex, corroborating previous findings [33, 38]. In fact, male individuals 


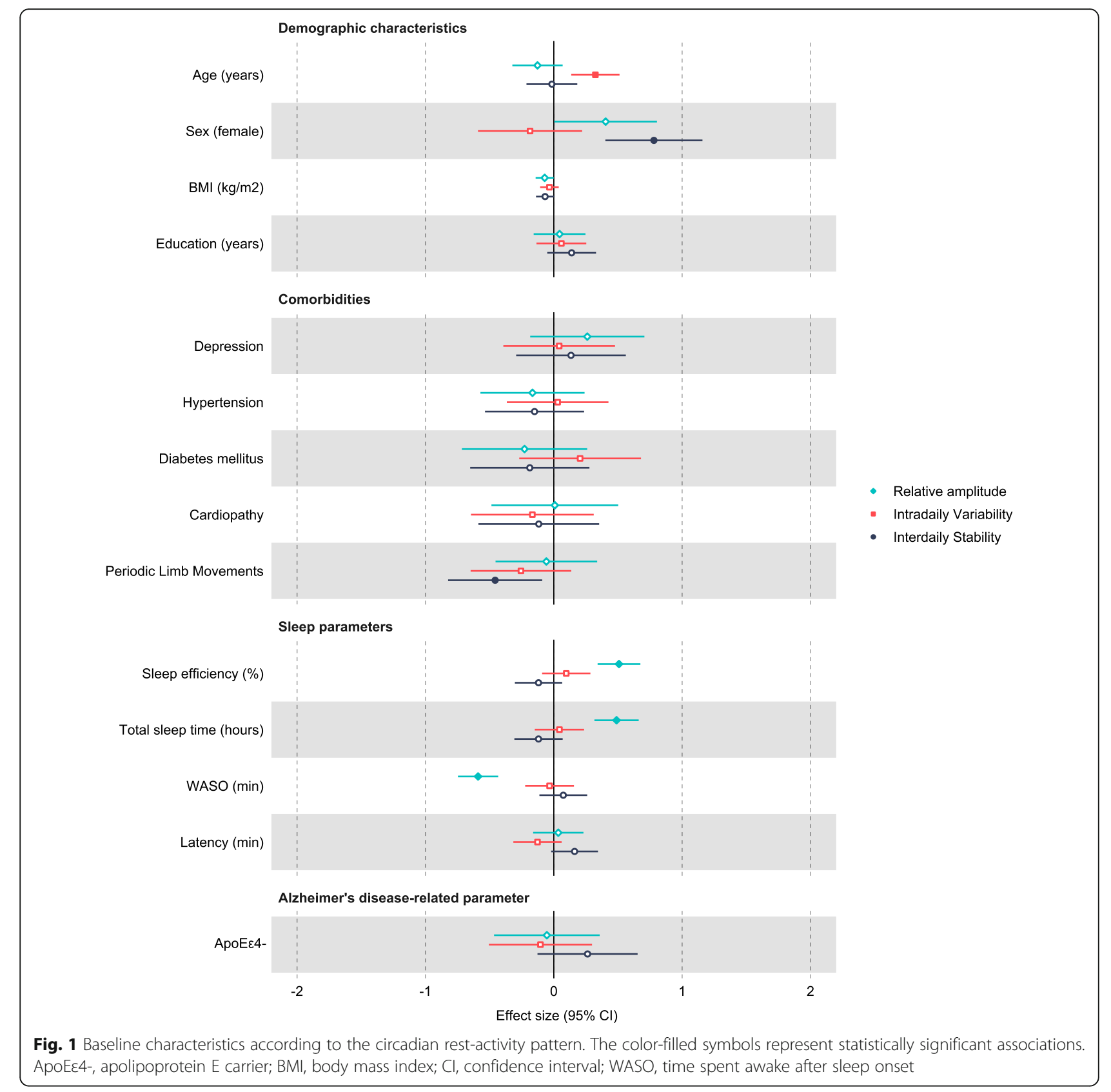

appear to be more vulnerable to circadian desynchronization induced by inappropriate light exposure [38], which is frequent among AD patients [39].

A previous study demonstrated increased fragmentation of the rest-activity rhythm in cognitively normal individuals with preclinical amyloid plaque pathology, indicating that circadian dysfunction could be a marker for the preclinical stage and/or contribute to the pathogenesis of the disease [10]. Accordingly, healthy older adults with lower amplitude and higher fragmentation of the rhythm demonstrated an increased risk of developing Alzheimer's dementia [8]. Additionally, individuals with mild cognitive impairment and high intradaily variability presented an increased risk of conversion to $A D$ compared with those with lower fragmentation [8]. Here, we demonstrated that increased intradaily variability at baseline was associated with greater cognitive decline after one year of follow-up, especially in males. This suggests a predictive role of the rest-activity pattern for cognition once individuals are already diagnosed with $\mathrm{AD}$. Although the relationship between circadian function and disease progression appears to be bidirectional [8, $12,40]$, some studies suggest mechanisms by which circadian alterations could foster cognitive decline. High 
Table 3 CSF biomarkers according to the circadian rest-activity pattern

\begin{tabular}{|c|c|c|c|c|c|c|}
\hline \multirow[t]{2}{*}{ Biomarkers } & \multicolumn{2}{|l|}{ Intradaily variability } & \multicolumn{2}{|l|}{ Interdaily stability } & \multicolumn{2}{|l|}{ Relative amplitude } \\
\hline & Effect size $(95 \%$ CI) & $p$ value & Effect size $(95 \%$ Cl) & $p$ value & Effect size $(95 \%$ CI) & $p$ value \\
\hline \multicolumn{7}{|l|}{$A \beta 42$} \\
\hline Global & $0.067(-0.144$ to 0.277$)$ & 0.529 & $-0.177(-0.391$ to 0.038$)$ & 0.106 & $-0.192(-0.405$ to 0.020$)$ & 0.076 \\
\hline$A \beta 42+$ & $-0.150(-0.337$ to 0.037$)$ & 0.114 & $-0.042(-0.231$ to 0.147$)$ & 0.660 & $-0.010(-0.195$ to 0.175$)$ & 0.910 \\
\hline \multicolumn{7}{|l|}{$T$-tau } \\
\hline Global & $-0.159(-0.367$ to 0.050$)$ & 0.133 & $0.109(-0.109$ to 0.326$)$ & 0.322 & 0.343 (0.139 to 0.547$)$ & 0.001 \\
\hline$A \beta 42+$ & $-0.034(-0.315$ to 0.247$)$ & 0.809 & $0.172(-0.102$ to 0.445$)$ & 0.213 & 0.304 (0.046 to 0.561$)$ & 0.022 \\
\hline \multicolumn{7}{|l|}{ P-tau } \\
\hline Global & $-0.089(-0.300$ to 0.122$)$ & 0.404 & $0.068(-0.151$ to 0.286$)$ & 0.540 & $0.205(-0.008$ to 0.418$)$ & 0.059 \\
\hline$A \beta 42+$ & $0.071(-0.228$ to 0.370$)$ & 0.636 & $0.095(-0.200$ to 0.390$)$ & 0.521 & $0.114(-0.174$ to 0.401$)$ & 0.431 \\
\hline \multicolumn{7}{|l|}{$N F-L$} \\
\hline Global & $-0.056(-0.298$ to 0.185$)$ & 0.642 & 0.322 (0.076 to 0.569$)$ & 0.011 & $0.444(0.212$ to 0.676$)$ & 0.001 \\
\hline$A \beta 42+$ & $-0.070(-0.389$ to 0.250$)$ & 0.662 & $0.309(-0.003$ to 0.620$)$ & 0.052 & 0.503 (0.209 to 0.797 ) & 0.001 \\
\hline
\end{tabular}

Linear regression models adjusted by age, sex, and season of the year were performed to assess the biomarkers levels according to the circadian rest-activity pattern

Aß42 amyloid-beta protein, $C l$ confidence interval, NF-L neurofilament light, $P$-tau phosphorylated-tau, $T$-tau total-tau

fragmentation of the rest-activity rhythm is a consequence of increased periods of rest during the active phase and/or increased periods of activity during the rest phase, which ultimately affects sleep quality. Sleep is an important process for memory consolidation [41] and sleep deprivation or pharmacologically induced wakefulness increase the levels of $A \beta 42$ [42, 43]. Thus, the increased cognitive decline in patients with higher fragmentation of the rest-activity rhythm could be, at least in part, a consequence of inappropriate sleep quality. Furthermore, a significant number of molecules associated with memory consolidation processes and with the pathophysiology of $\mathrm{AD}$ are under circadian control. Considering this, it is plausible to expect the fragmentation of the rest-activity rhythm to influence the cognitive decline $[44,45]$. Further studies will be necessary to investigate whether a relationship of causality between the circadian rest-activity pattern and cognitive decline is present and the possible mechanisms underlying this. Also, further evaluations will be necessary to confirm the possible differential effect of the sex within this context.

Our results showed an unexpected association between the amplitude of the rhythm and biomarkers such as T-tau and NF-L [46-48]. The relative amplitude represents the robustness of the rest-activity rhythm; hence, one would expect decreased levels of these biomarkers in patients with higher amplitude. In this regard, it is important to consider that both events could be simultaneously occurring as consequences of different processes without any causality between them. Additionally, these findings should be interpreted considering the

Table 4 Cognitive decline according to the circadian rest-activity pattern

\begin{tabular}{|c|c|c|c|c|}
\hline & \multicolumn{2}{|l|}{ Model 1} & \multicolumn{2}{|l|}{ Model 2} \\
\hline & Effect size $(95 \% \mathrm{Cl})$ & $p$ value & Effect size $(95 \% \mathrm{Cl})$ & $p$ value \\
\hline \multicolumn{5}{|c|}{ Intradaily variability } \\
\hline Global & $-0.665(-1.190$ to -0.140$)$ & 0.014 & $-0.715(-1.272$ to -0.157$)$ & 0.013 \\
\hline$A \beta 42+$ & $-0.522(-1.147$ to 0.103$)$ & 0.100 & $-0.717(-1.391$ to -0.042$)$ & 0.038 \\
\hline \multicolumn{5}{|c|}{ Interdaily stability } \\
\hline Global & $-0.266(-0.818$ to 0.286$)$ & 0.341 & -0.205 ( -0.817 to 0.407$)$ & 0.507 \\
\hline$A \beta 42+$ & -0.108 (- 0.778 to 0.562$)$ & 0.747 & 0.009 (- 0.690 to 0.708$)$ & 0.979 \\
\hline \multicolumn{5}{|c|}{ Relative amplitude } \\
\hline Global & $-0.495(-1.025$ to 0.036$)$ & 0.067 & $-0.587(-1.192$ to 0.019$)$ & 0.057 \\
\hline$A \beta 42+$ & $-0.658(-1.278$ to -0.038$)$ & 0.038 & $-0.520(-1.202$ to 0.163$)$ & 0.132 \\
\hline
\end{tabular}

Linear regression models were performed to assess the cognitive decline according to the circadian rest-activity pattern. Model 1 , unadjusted analysis; model 2 , adjusted for age, sex, T-tau/A 342 ratio, educational level, and season of the year Aß42 amyloid-beta protein, $\mathrm{Cl}$ confidence interval, $T$-tau total-tau 


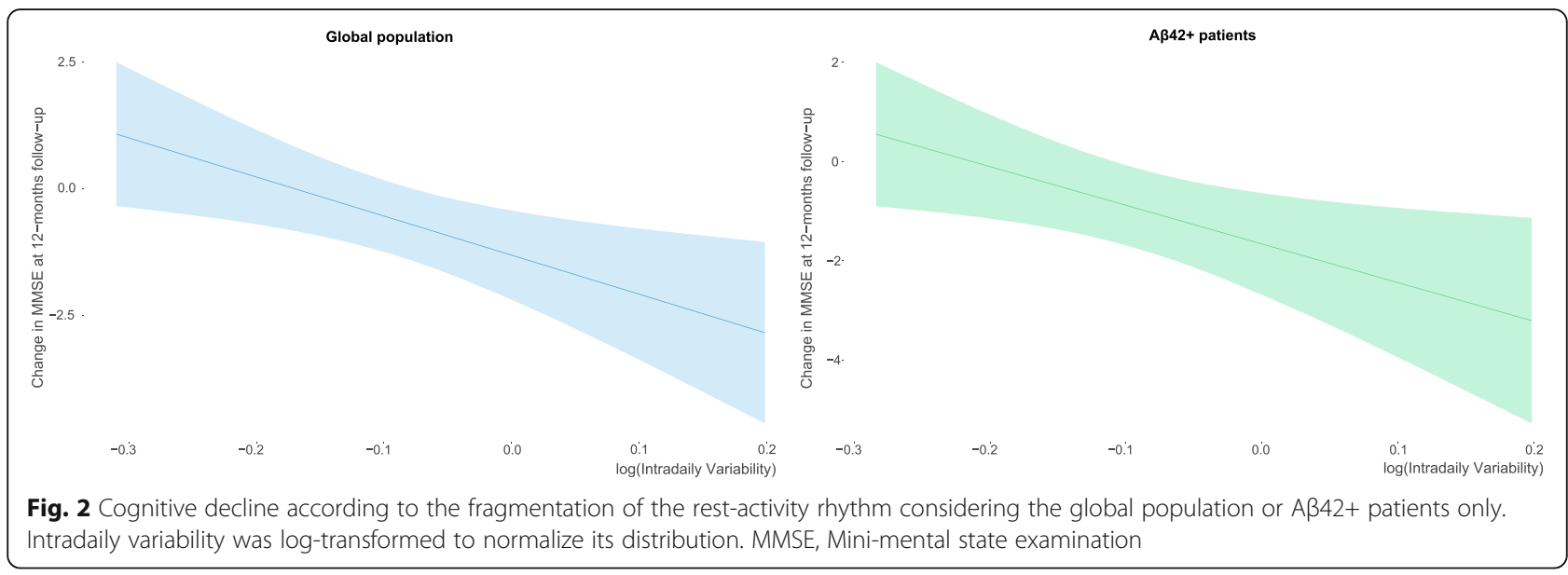

particularities of this population. A higher level of activity in AD patients during the day (contributing to higher amplitude) may indicate increased agitation and mental confusion, which is expected in more advanced cases with supposedly higher levels of T-tau and NF-L [48, 49]. Also, the observed association between NF-L and interdaily stability should be pondered. Institutionalized individuals are assigned to external schedules, which may wrongly suggest good stability of the rhythm $[8$, 39]. Even though none of the patients were institutionalized neither at baseline nor after one year of follow-up, they may be under the influence of relatives' schedules at an early stage of the disease. Further studies with a prospective design and with molecular markers of circadian function will be necessary to improve our understanding of this matter.

\section{Limitations}

The first limitation of this study is that the patients were enrolled from a cognitive unit, and not from a population-based community. Second, the observed associations between circadian rest-activity pattern and cognitive decline were exclusively based on the MMSE, preventing a detailed identification of the relevant cognitive domains in this regard. Third, given the low prevalence of males in our sample, the findings suggesting a differential effect of the sex on the investigated associations should be considered with caution and confirmed in future studies. Fourth, considering the observational design of this study, it is not possible to establish directionality between the associations of interest. Fifth, the measurements performed with actigraphy are influenced by physical activity. Other circadian markers in addition to the rest-activity rhythm should be considered to confirm our findings in future studies. Finally, it is important to address that only patients with mild-moderate $\mathrm{AD}$ were included in the analyses and therefore the results herein presented should be limited to this specific population. The observed associations might change according to the stage of the disease or contexts other than $\mathrm{AD}$.

\section{Conclusions}

In summary, we observed that older age and male sex were associated with worse outcomes in terms of restactivity patterns in a population of mild-moderate $\mathrm{AD}$ patients. Accordingly, older age was associated with increased fragmentation of the rhythm, while male sex was related to lower synchronization between the endogenous rhythm and the zeitgebers. In addition, increased fragmentation of the rhythm at baseline was associated with greater cognitive decline after one year of followup. This finding sets the rest-activity pattern as a potential predictor of cognitive decline in mild-moderate $\mathrm{AD}$ patients. Further studies are warranted to confirm our findings and to improve our understanding of the possible association between the rest-activity pattern and CSF biomarkers once the individuals are diagnosed with the disease.

\section{Abbreviations}

AD: Alzheimer's disease; ApoE: Apolipoprotein E; Aß42: Amyloid-beta protein; BMI: Body mass index; Cl: Confidence interval; CSF: Cerebrospinal fluid; MMSE: Mini-Mental State Examination; NF-L: Neurofilament light; Ptau: Phosphorylated-tau; SE: Standard error of mean; T-tau: Total tau; WASO: Wake after sleep onset

\section{Supplementary Information}

The online version contains supplementary material available at https://doi. org/10.1186/s13195-021-00903-7.

Additional file 1 : Suppl Table 1. Baseline characteristics of $A \beta 42+$ and Aß42- patients.

Additional file $\mathbf{2}$ : Suppl Table $\mathbf{2}$. Circadian rest-activity pattern and sleep of $A \beta 42+$ and $A \beta 42$ - patients.

Additional file 3 : Suppl Table 3. CSF biomarkers according to the circadian rest-activity pattern in A 342 - patients. 
Additional file 4 : Suppl Table 4. CSF biomarkers according to the circadian rest-activity pattern in males and females.

Additional file 5 : Suppl Table 5. Cognitive decline according to the circadian rest-activity pattern in A 442 - patients.

Additional file $\mathbf{6}$ : Suppl Figure 1. Cognitive decline according to the fragmentation of the rest-activity rhythm in A 442 - patients.

Additional file 7 : Suppl Table 6. Cognitive decline according to the circadian rest-activity pattern in males and females.

\section{Acknowledgements}

We would like to express our sincere gratitude to all of the patients, members of the Sleep and Dementia Unit at the Hospital Universitari Santa Maria, and Yo-El Ju for the critical revision of the manuscript. We were supported by the IRBLleida Biobank (B.0000682) and PLATAFORMA BIOBANCOS PT17/0015/0027/.

\section{Authors' contributions}

$A T, I B, F B$, and GPR designed the study; AT, IB, and JFA searched the literature; FD, OM, HZ, KB, and GPR collected the data; IB and AT analyzed the data; AT, IB, JFA, and GPR interpreted the data; AT wrote the manuscript draft. All authors revised the manuscript and approved it for submission.

\section{Funding}

Generalitat of Catalonia, Department of Health (PERIS 2019 SLT008/18/00050) and "Fundació La Marató TV3" (464/C/2014) to GPR. Co-financed by FEDER funds from the European Union ('A way to build Europe'). IRBLleida is a CERCA Programme/Generalitat of Catalonia. FD was supported by Agency for Management of University and Research Grants (FI_B100153).

\section{Availability of data and materials}

The datasets used and/or analyzed during the current study are available from the corresponding author on reasonable request.

\section{Declarations}

\section{Ethics approval and consent to participate}

The study was approved by the care ethics committee of Hospital Universitari Arnau de Vilanova (CE-1218) and conducted according to the Declaration of Helsinki. The patient, the responsible caregiver, and the legal representative (when different from the responsible caregiver) signed an informed consent form.

\section{Consent for publication}

Not applicable.

\section{Competing interests}

The authors declare that they have no competing interests.

\section{Author details}

${ }^{1}$ Translational Research in Respiratory Medicine, Hospital Universitari Arnau de Vilanova-Santa Maria, IRBLleida, Lleida, Spain. ${ }^{2}$ Centro de Investigación Biomédica en Red de Enfermedades Respiratorias (CIBERES), Madrid, Spain. ${ }^{3}$ Unitat Trastorns Cognitius, Clinical Neuroscience Research, Hospital Universitari Santa Maria, IRBLleida, Lleida, Spain. ${ }^{4}$ Department of Physiology and Behavior, Federal University of Rio Grande do Norte, Natal, Brazil. ${ }^{5}$ Department of Molecular Neuroscience, UCL Institute of Neurology, Queen Square, London, UK. ${ }^{6}$ UK Dementia Research Institute, London, UK. ${ }^{7}$ Institute of Neuroscience and Physiology, Department of Psychiatry and Neurochemistry, University of Gothenburg, Sahlgrenska University Hospital, Mölndal, Sweden. ${ }^{8}$ Clinical Neurochemistry Laboratory, Sahlgrenska University Hospital, Mölndal, Sweden.

Received: 23 June 2021 Accepted: 12 September 2021

\section{Published online: 25 September 2021}

\section{References}

1. Prince M, Bryce R, Albanese E, Wimo A, Ribeiro W, Ferri CP. The global prevalence of dementia: a systematic review and metaanalysis. Alzheimers Dement. 2013;9(1):63-75.e2. https://doi.org/10.1016/j.jalz.2012.11.007.
2. Nichols E, Szoeke CEl, Vollset SE, Abbasi N, Abd-Allah F, Abdela J, et al. Global, regional, and national burden of Alzheimer's disease and other dementias, 1990-2016: a systematic analysis for the Global Burden of Disease Study 2016. Lancet Neurol. 2019;18(1):88-106. https://doi.org/10.101 6/S1474-4422(18)30403-4.

3. United Nations. World Population Ageing 2019 Highlights. World Popul. Ageing 2019 Highlights. 2019. https://doi.org/10.18356/9df3caed-en.

4. Alzheimer's disease facts and figures. Alzheimer's Dement. 2020. https://doi. org/10.1002/alz.12068.

5. Masters $\mathrm{CL}$, Bateman R, Blennow K, Rowe CC, Sperling RA, Cummings JL. Alzheimer's disease. Nat Rev Dis Primers. 2015;1:15056 Available from: http:// www.nature.com/articles/nrdp201556.

6. Caraci F, Copani A, Nicoletti F, Drago F. Depression and Alzheimer's disease: Neurobiological links and common pharmacological targets. Eur J Pharmacol. 2010;626(1):64-71. https://doi.org/10.1016/j.ejphar.2009.10.022.

7. Peter-Derex L, Yammine P, Bastuji H, Croisile B. Sleep and Alzheimer's disease. Sleep Med Rev. 2015;19:29-38. https://doi.org/10.1016/j.smrv.2014. 03.007.

8. Li P, Gao L, Gaba A, Yu L, Cui L, Fan W, et al. Circadian disturbances in Alzheimer's disease progression: a prospective observational cohort study of community-based older adults. Lancet Heal Longev. 2020;7568:1-10 The Author(s). Published by Elsevier Ltd. This is an Open Access article under the CC BY-NC-ND 4.0 license.

9. Ancoli-Israel S, Klauber MR, Jones DW, Kripke DF, Martin J, Mason W, et al. Variations in circadian rhythms of activity, sleep, and light exposure related to dementia in nursing-home patients. Sleep. 1997;20:18-23.

10. Musiek ES, Bhimasani M, Zangrilli MA, Morris JC, Holtzman DM, Ju YES. Circadian rest-activity pattern changes in aging and preclinical Alzheimer disease. JAMA Neurol. 2018;75(5):582-90.

11. Duffy JF, Zitting KM, Chinoy ED. Aging and circadian rhythms. Sleep Med Clin. 2015;10(4):423-34.

12. Leng Y, Musiek ES, Hu K, Cappuccio FP, Yaffe K. Association between circadian rhythms and neurodegenerative diseases. Lancet Neurol. 2019; 18(3):307-18.

13. Wang JL, Lim AS, Chiang WY, Hsieh WH, Lo MT, Schneider JA, et al. Suprachiasmatic neuron numbers and rest-activity circadian rhythms in older humans. Ann Neurol. 2015;78(2):317-22.

14. Putilov A. Age-associated Advance of Sleep Times Relative to the Circadian Phase of Alertness-sleepiness Rhythm: Can it be Explained by Changes in Ratios Between Strengths of the Underlying Oscillatory Processes? Curr Aging Sci. 2016;9(1):44-56.

15. Weissová K, Bartoš A, Sládek M, Nováková M, Sumová A. Moderate changes in the circadian system of Alzheimer's disease patients detected in their home environment. PLoS One. 2016;11(1):e0146200.

16. Okawa M, Mishima K, Hishikawa Y, Hozumi S, Hori H, Takahashi K. Circadian rhythm disorders in sleep-waking and body temperature in elderly patients with dementia and their treatment. Sleep. 1991;14(6):478-85.

17. Hatfield CF, Herbert J, Van Someren EJW, Hodges JR, Hastings MH. Disrupted daily activity/rest cycles in relation to daily cortisol rhythms of home-dwelling patients with early Alzheimer's dementia. Brain. 2004;127(5): 1061-74. https://doi.org/10.1093/brain/awh129.

18. Walsh CM, Blackwell T, Tranah GJ, Stone KL, Ancoli-Israel S, Redline S, et al. Weaker circadian activity rhythms are associated with poorer executive function in older women. Sleep. 2014;37(12):2009-16. https://doi.org/10. 5665/sleep.4260.

19. Tranah GJ, Blackwell T, Stone KL, Ancoli-Israel S, Paudel ML, Ensrud KE, et al. Circadian activity rhythms and risk of incident dementia and mild cognitive impairment in older women. Ann Neurol. 2011;70(5):722-32. https://doi. org/10.1002/ana.22468.

20. Schlosser Covell GE, Dhawan PS, Lee lannotti JK, Hoffman-Snyder CR, Wellik KE, Caselli RJ, et al. Disrupted daytime activity and altered sleep-wake patterns may predict transition to mild cognitive impairment or dementia: a critically appraised topic. Neurologist. 2012;18(6):426-9. https://doi.org/10.1 097/NRL.0b013e318272f7ef.

21. Rogers-Soeder TS, Blackwell T, Yaffe K, Ancoli-Israel S, Redline S, Cauley JA, et al. Rest-Activity Rhythms and Cognitive Decline in Older Men: The Osteoporotic Fractures in Men Sleep Study. J Am Geriatr Soc. 2018;66(11): 2136-43. https://doi.org/10.1111/jgs.15555.

22. McKhann GM, Knopman DS, Chertkow H, Hyman BT, Jack CR, Kawas CH, et al. The diagnosis of dementia due to Alzheimer's disease: Recommendations from the National Institute on Aging-Alzheimer's 
Association workgroups on diagnostic guidelines for Alzheimer's disease. Alzheimers Dement. 2011;7(3):263-9. https://doi.org/10.1016/j.jalz.2011.03. 005 .

23. Targa A, Dakterzada F, Benítez ID, de Gonzalo-Calvo D, Moncusí-Moix A, López R, et al. Circulating MicroRNA profile associated with obstructive sleep apnea in alzheimer's disease. Mol Neurobiol. 2020;57(11):4363-72.

24. Gonçalves BSB, Adamowicz T, Louzada FM, Moreno CR, Araujo JF. A fresh look at the use of nonparametric analysis in actimetry. Sleep Med Rev. 2015; 20:84-91.

25. Witting W, Kwa IH, Eikelenboom P, Mirmiran M, Swaab DF. Alterations in the circadian rest-activity rhythm in aging and Alzheimer's disease. Biol Psychiatry. 1990;27(6):563-72.

26. Folstein MF, Folstein SE, McHugh PR, Fanijang G. MMSE - Examen Cognoscitivo Mini-mental. J Psychiatr Res. 1975;12(3):189-98. Madrid: TEA Ediciones. Available from: https://linkinghub.elsevier.com/retrieve/pii/00223 95675900266. https://doi.org/10.1016/0022-3956(75)90026-6.

27. Folstein MF, Folstein SE, McHugh PR. "Mini-mental state". A practical method for grading the cognitive state of patients for the clinician. J Psychiatr Res. 1975;12(3):189-98.

28. Tukey JW. Exploratory data analysis by John W. Tukey. Biometrics. 1977. https://doi.org/10.1007/978-0-387-39940-9_1384.

29. Lim ASP, Gaiteri C, Yu L, Sohail S, Swardfager W, Tasaki S, et al. Seasonal plasticity of cognition and related biological measures in adults with and without Alzheimer disease: Analysis of multiple cohorts. PLoS Med. 2018; 15(9):e1002647. https://doi.org/10.1371/journal.pmed.1002647.

30. Gao L, Li P, Gaba A, Musiek E, Ju YS, Hu K. Fractal motor activity regulation and sex differences in preclinical Alzheimer's disease pathology. Alzheimer's Dement Diagnosis, Assess Dis Monit. 2021;13:1-9.

31. Carrier J, Semba K, Deurveilher S, Drogos L, Cyr-Cronier J, Lord C, et al. Sex differences in age-related changes in the sleep-wake cycle. Front Neuroendocrinol. 2017;47:66-85. Elsevier; Available from:. https://doi.org/1 0.1016/j.yfrne.2017.07.004.

32. Jack CR, Bennett DA, Blennow K, Carrillo MC, Dunn B, Haeberlein SB, et al. NIA-AA research framework: toward a biological definition of Alzheimer's disease. Alzheimers Dement. 2018;14(4):535-62.

33. Mitchell JA, Quante M, Godbole S, James P, Hipp JA, Marinac CR, et al. Variation in actigraphy-estimated rest-activity patterns by demographic factors. Chronobiol Int. 2017;34:1042-56. Taylor \& Francis; .Available from: https://doi.org/10.1080/07420528.2017.1337032.

34. Luik Al, Zuurbier LA, Hofman A, Van Someren EJW, Tiemeier H. Stability and fragmentation of the activity rhythm across the sleep-wake cycle: the importance of age, lifestyle, and mental health. Chronobiol Int. 2013;30(10): 1223-30. https://doi.org/10.3109/07420528.2013.813528.

35. Kadono M, Nakanishi N, Yamazaki M, Hasegawa G, Nakamura N, Fukui M. Various patterns of disrupted daily rest-activity rhythmicity associated with diabetes. J Sleep Res. 2016;25(4):426-37. https://doi.org/10.1111/jsr.12385.

36. Rogers VE, Zhu S, Mandrell BN, Ancoli-Israel S, Liu L, Hinds PS. Relationship between circadian activity rhythms and fatigue in hospitalized children with CNS cancers receiving high-dose chemotherapy. Support Care Cancer. Support Care Cancer. 2020;28(3):1459-67. https://doi.org/10.1007/s00520-01 9-04960-5.

37. Jeon S, Conley S, Redeker NS. Rest-activity rhythms, daytime symptoms, and functional performance among people with heart failure. Chronobiol Int. 2020;37:1223-34. Taylor \& Francis; Available from:. https://doi.org/10.1080/ 07420528.2020.1779280.

38. Müch M, Schmieder M, Bieler K, Goldbach R, Fuhrmann T, Zumstein N, et al. Bright Light Delights: Effects of Daily Light Exposure on Emotions, Restactivity Cycles, Sleep and Melatonin Secretion in Severely Demented Patients. Curr Alzheimer Res. 2017;14(10):1063-75. https://doi.org/10.2174/1 567205014666170523092858

39. Van Someren EJW, Hagebeuk EEO, Lijzenga C, Scheltens P, SEJA DR, Jonker C, et al. Circadian rest-activity rhythm disturbances in Alzheimer's disease. Biol Psychiatry. 1996;40(4):259-70

40. Uddin MS, Tewari D, Mamun AA, Kabir MT, Niaz K, MII W, et al. Circadian and sleep dysfunction in Alzheimer's disease. Ageing Res Rev. 2020;60 101046. Elsevier; Available from:. https://doi.org/10.1016/j.arr.2020.101046.

41. Rasch B. About sleep's role in memory. Psychologist. 2014;93(2):681-766.

42. Shokri-Kojori E, Wang GJ, Wiers CE, Demiral SB, Guo M, Kim SW, et al. $\beta$ Amyloid accumulation in the human brain after one night of sleep deprivation. Proc Natl Acad Sci U S A. 2018;115(17):4483-8.
43. Kang J-E, Lim MM, Bateman RJ, Lee JJ, Smyth LP, Cirrito JR, et al. Orexin and the Sleep-Wake Cycle. Science (80- ). 2009;326:1005-8.

44. Aten S, Hansen KF, Snider K, Wheaton K, Kalidindi A, Garcia A, et al. miR-132 couples the circadian clock to daily rhythms of neuronal plasticity and cognition. Learn Mem. 2018;25(5):214-29. https://doi.org/10.1101/Im.0471 91.117.

45. Heyde I, Kiehn JT, Oster H. Mutual influence of sleep and circadian clocks on physiology and cognition. Free Radic Biol Med. 2018;119:8-16. Elsevier BV; Available from:. https://doi.org/10.1016/j.freeradbiomed.2017.11.003.

46. Bridel C, van Wieringen WN, Zetterberg H, Tijms BM, Teunissen CE, AlvarezCermeño JC, et al. Diagnostic value of cerebrospinal fluid neurofilament light protein in neurology. JAMA Neurol. 2019;76:1035 Available from: https://jamanetwork.com/journals/jamaneurology/fullarticle/2735955.

47. Alcolea D, Vilaplana E, Suárez-Calvet M, Illán-Gala I, Blesa R, Clarimón J, et al. CSF SAPP $B, Y K L-40$, and neurofilament light in frontotemporal lobar degeneration. Neurology. 2017;89(2):178-88. https://doi.org/10.1212/WNL. 0000000000004088

48. Preische O, Schultz SA, Apel A, Kuhle J, Kaeser SA, Barro C, et al. Serum neurofilament dynamics predicts neurodegeneration and clinical progression in presymptomatic Alzheimer's disease. Nat Med. 2019;25(2): 277-83. https://doi.org/10.1038/s41591-018-0304-3.

49. Rajan KB, Aggarwal NT, McAninch EA, Weuve J, Barnes LL, Wilson RS, et al. Remote blood biomarkers of longitudinal cognitive outcomes in a population study. Ann Neurol. 2020;88(6):1065-76. https://doi.org/10.1002/a na.25874.

\section{Publisher's Note}

Springer Nature remains neutral with regard to jurisdictional claims in published maps and institutional affiliations.
Ready to submit your research? Choose BMC and benefit from:

- fast, convenient online submission

- thorough peer review by experienced researchers in your field

- rapid publication on acceptance

- support for research data, including large and complex data types

- gold Open Access which fosters wider collaboration and increased citations

- maximum visibility for your research: over $100 \mathrm{M}$ website views per year

At $\mathrm{BMC}$, research is always in progress.

Learn more biomedcentral.com/submissions 